\title{
Comparison of coronary CT angiography with conventional coronary angiography in the diagnosis of coronary artery disease
}

\author{
Mannan $\mathrm{M}^{1}$, Bashar $\mathrm{MA}^{2}$, Mohammad J ${ }^{3}$, Jahan $\mathrm{MU}^{4}$, Momenuzzaman $\mathrm{NAM}^{5}$, Haque $\mathrm{MA}^{6}$ \\ ${ }^{1}$ Medinova Medical Services Ltd., Dhaka, ${ }^{2}$ Department of Radiology and Imaging, BIHS, Dhaka, \\ ${ }^{3}$ Department of Radiology and Imaging, United Hospital Ltd., Dhaka, ${ }^{4}$ Maternal and Child Health \\ Training Institute, Azimpur, Dhaka, ${ }^{5}$ Department of Cardiology, United Hospital Ltd., Dhaka, \\ ${ }^{6}$ Department of Public Health and Informatics, Bangabandhu Sheikh Mujib Medical University, \\ Dhaka.Email: maksudam27@yahoo.com
}

\begin{abstract}
Noninvasive CT coronary angiography is a promising coronary imaging technique. In spite of the unprecedented temporal and spatial resolution and the inability to perform therapeutic interventions in the same session multi-detector computed tomography (MDCT) has been considering a promising alternative, non invasive tool for coronary artery imaging due to its high sensitivity and specificity for the detection of significant coronary artery stenosis. To evaluate the diagnostic accuracy of 64-slice MDCT for assessing haemodynamically significant stenoses of the coronary arteries in comparison with the conventional standard cardiac angiography. Fifty patients scheduled for conventional coronary angiography at the department of Radiology and Imaging, United Hospital, Dhaka were enrolled between July 2007 and June 2008. All patients underwent both conventional and MDCT angiography within mean 10.70 days. Overall sensitivity of 64-slice MDCT for the detection of stenosis $\leq 50 \%$, stenosis $>50 \%$, and stenosis $>75 \%$ was $90.0 \%, 83.8 \%$, and $80.7 \%$, respectively, and specificity was $96.5 \%, 98.4 \%$, and $98.3 \%$ respectively and accuracy was $96.0 \%, 96.5 \%$, and $96.6 \%$ respectively. Contrast-enhanced 64-slice MDCT allows the identification of coronary stenosis with excellent accuracy. Measurements of stenosis derived by MDCT correlated well with conventional angiogram. A major limitation is the insufficient ability of CT to exactly quantify the degree of stenosis.
\end{abstract}

\section{Introduction}

Significant advancement in diagnostic technology using CT has appeared over the last decade. Though the conventional coronary angiography is considered as gold standard for the evaluation of coronary artery stenoses, MDCT is currently becoming more popular because of its non invasive nature.

The ability to visualize coronary artery lumen and wall, obtain information on the presence, severity and characteristics of coronary artery disease noninvasively, MDCT is a better diagnostic tool compare to others including nuclear perfusion imaging or invasive coronary angiography .

With remarkable improvement reported on the diagnostic accuracy with 64 slice MDCT, many limitations remained, hindering the application of this promising noninvasive technology as a diagnostic tool in the routine clinical workup of patients with coronary artery disease (CAD). These limitations included inability to explain small coronary artery branches less than $2 \mathrm{~mm}$, cardiac motion artifacts in fast or arrhythmic heart rates, and partial voluming effects of severe calcium deposits in the coronary artery wall. These render a significant number of scans or vessel segments incompletely interpretable ${ }^{2-4}$. Initial reports on MDCT are very promising, reporting a sensitivity and specificity for the detection of significant stenoses of $94 \%$ and $97 \%$, respectively ${ }^{5}$. Thus, the aim of the present study was to determine the diagnostic accuracy of a 64-slice MDCT for assessing haemodynamically significant ( $\geq 70 \%$ stenosis) or non significant $(<70 \%$ stenosis $)$ stenoses of the coronary arteries in comparison with the conventional gold standard cardiac angiography. This was done on both per patient and per segment analyses.

\section{Materials and Methods}

It was a cross sectional type of non-randomized, efficacy study. Patients who were attended in United Hospital, Dhaka for both conventional angiography and CT angiography for their proper management were selected for this study. The investigators interpreting the MDCT results were blinded to the results of the angiogram and vice versa.

Sample size was determined by power analysis for a single proportion. We hypothesized that 64 slice MDCT should detect greater than 50\% lumen narrowing, with a sensitivity of $80 \%$ or greater. The sample size was calculated for a power level of greater than $80 \%$, an $\alpha$ error of 0.05 , and an expected sensitivity of $94 \%$ or greater based on previous reports. 
Inclusion criteria: Patients of both sexes aged 18 years and above.

Exclusion criteria: Patients with Coronary artery bypass graft $(\mathrm{CABG})$ or having stent in coronary artery; atrial fibrillation; obstructive pulmonary disease; severe peripheral vascular disease; sick sinus syndrome or AV block greater than $1^{\text {st }}$ degree; renal disease or other conditions that might increase the risk of contrast nephropathy; systolic blood pressure below $100 \mathrm{~mm}$ of $\mathrm{Hg}$; hypothyroidism (thyrotropin level $<0.44 \mathrm{mIU} / \mathrm{L}$ ); unstable clinical condition (like acute conronary syndrome); patients contraindicated for beta blockers and iodine containing contrast agents.

Oral administration of $50 \mathrm{mg}$ of metoprolol (50mg) was given in patients who had heart rates between 60-70 beats/minute 60 minutes prior to the scheduled CT scan and $100 \mathrm{mg}$ metoprolol in case of $>70$ beats/minute.

Computed topographic angiography was performed using a 64-slice MDCT scanner. A bolus of 1.2 $\mathrm{ml} / \mathrm{kg} \mathrm{ml}$ of contrast agent (Iopamiro $370 \mathrm{mg} / \mathrm{ml}$, omnipaque $350 \mathrm{mg} / \mathrm{m}$ ) was injected intravenously (4.5 $\mathrm{ml}-6 \mathrm{ml} / \mathrm{sec})$. As soon as the signal in the ascending aorta was reached a predefined threshold of 120-150 HU, the scan was started automatically and the entire volume of the heart was acquired during one breath hold in 6-10 s with simultaneous recording of the electrocardiographic trace.

The CT scan was analyzed by two independent radiologists and consensus was made by third radiologists. In a first step, image quality was determined by the investigators on the basis of the presence of motion artifacts and on the basis of the contrast-to-noise ratio.

The grading criteria for image quality was as follows as described by Leber et al ${ }^{6}$ : A high-quality image is defined as no motion artifacts and a contrast-to-noise ratio of $>8$; a moderate-quality image is defined as motion artifacts present but the vessel still evaluable or a contrast-to-noise ratio between 4 and 8 ; and a poor-quality image is defined as motion artifacts present that made vessel delineation impossible or a contrast-to noise ratio $<4$. Only patients who had high- and moderate quality images of all coronary segments were considered for further analysis.

Non calcified coronary atherosclerotic plaque was defined as any noticeable structure attached to the coronary artery wall having CT density less than the contrast-enhanced coronary lumen but greater than the surrounding connective tissue.

Calcified coronary atherosclerotic plaque was defined as any assigned structure in coronary artery lumen with a density of $130 \mathrm{HU}$ or more, visualized separately from the contrast-enhanced coronary lumen. This plaque should be identified in at least two independent planes ${ }^{7}$.

For comparison with conventional coronary angiography (ICA), the grade of diameter stenosis (maximum diameter reduction) was determined in longitudinal curved multiplanar reformatted reconstructions by dividing the minimal diameter in the diseased segment through the diameter in the adjacent proximal disease-free section in the same two projections that was used for ICA.

\section{Conventional coronary angiography}

Standard procedure was followed in performing conventional coronary angiography. Angiograms were evaluated by an experienced cardiologist who was blinded to the results from previous CT coronary angiography. The coronary arteries were segmented according to the guidelines of the American Heart Association ${ }^{\mathbf{8}}$ as follows: the right coronary artery (RCA) was subdivided in a proximal, middle, and distal parts; the posterior descending artery was considered as independent because of the variability of its origin; the left circumflex artery (LCX) was divided into proximal and distal parts; the left anterior descending artery (LAD) was divided into proximal, middle, and distal parts; the diagonal (DIA) branches, obtuse marginal (OM) branches, and posterolateral (PLA) branches were considered as being independent segments. Each vessel segment was scored as being stenosed $\leq 50 \%,>50 \%$, and $\geq 75 \%$. Coronary artery analysis was performed in all vessels with a diameter down to $1.5 \mathrm{~mm}$, including those vessels distal to complete occlusions

Statistical analysis: For the statistical analysis, one Microsoft Windows-based software products was used (SPSS 12) for Windows, SPPS Incorporation, Chicago, IL, USA).

Diagnostic accuracy of 64 slice MDCT were calculated for stenosis $\geq 75 \%$, for stenosis $>50 \%$, and for lesions $\leq 50 \%$ diameter reduction, according to the conventional coronary angiography results. Sensitivity, specificity, diagnostic accuracy, negative predictive value (NPV), and positive predictive value (PPV) were calculated per patient and per segment of the vessels.

The interobserver agreement for the detection and exclusion of coronary artery stenosis for the two radiologists were quantified using the kappa value and interpreted as follows: less than 0.20 , poor agreement; $0.21-0.40$, fair agreement; $0.41-0.60$, moderate agreement; $0.61-0.80$, good agreement; and $0.81-1.00$, very good agreement. We took the consensus result for final evaluation. 


\section{Results}

The ethical committee of the Bangladesh College of Physicians and Surgeons, Dhaka, approved the study protocol, and all patients gave written informed consent. The patients mean age was $54.06 \pm 9.78$ years. There were $37 \mathrm{men}$, and 13 women. Their average weight was $69.78 \pm 9.86 \mathrm{~kg}$. The study protocol included the oral administration of $50 \mathrm{mg}$ of metoprolol 60 minutes before the scheduled CT scan in $68.0 \%$ patients with heart rates between 60 to 70 beats $/ \mathrm{min}$ and $100 \mathrm{mg}$ metoprolol in $22.0 \%$ patients in case of $>70$ beats/min.

Total of 850 segments of coronary artery were evaluated. Of all segments observer 1 evaluated $77.9 \%$ as normal or not detectable stenosis, $9.8 \%$ $\leq 50 \%$ stenosis, $3.1 \% 50 \%$ to $75 \%$ and $9.3 \%>75 \%$ stenosis. By observer 2 seventy eight percent segments were evaluated as normal or not detectable stenosis, $9.8 \%$ up to $50 \%$ stenosis, $2.9 \%$ above $50 \%$ to $75 \%$ stenosis and $9.3 \%$ above $75 \%$ stenosis. In consensus $77.1 \%$ had normal or not detectable, $10.6 \%$ had up to $50 \%$ stenosis, $2.9 \%$ above $50 \%$ to $75 \%$ and $9.4 \%$ had above $75 \%$ stenosis.

By cardiologists working in the department of cardiology of same hospital $78.7 \%$ segments were evaluated as normal or not detectable narrowing, $8.2 \%$ had up to $50 \%$ stenosis, $3.3 \%$ had $>50$ to $75 \%$ stenosis and $9.8 \%$ had above $75 \%$ stenosis. High levels of sensitivities, specificities, PPVs, NPVs and accuracies were observed in the detection of coronary artery stenosis by 64 slice MDCT.

Diagnostic accuracy of MDCT in detecting $\leq 50 \%$ stenoses, $>50 \%$ stenoses, and $>75 \%$ stenoses in coronary arteries are depicted in Table II and overall accuracies were shown in Table III.

Table I: Patients characteristics $(\mathrm{n}=50)$

\begin{tabular}{ll}
\hline Characteristics & Value \\
\hline Male sex, n (\%) & $37(74.0)$ \\
Age (yrs) (Mean \pm SD [range]) & $54.06 \pm 9.78$ [38-83] \\
Weight (kg) $($ Mean \pm SD) & $69.78 \pm 9.86$ \\
Pulse/minute (Mean \pm SD) & $68.36 \pm 13.04$ \\
Systolic BP mm of Hg (Mean \pm SD) & $136.30 \pm 22.85$ \\
Diastolic BP mm of Hg (Mean \pm SD) & $86.08 \pm 9.75$ \\
Heart rate/ minute (Mean \pm SD) & $66.78 \pm 6.13$ \\
Interval time between two interventions & $10.70 \pm 9.79[1-46]$ \\
(day) (Mean \pm SD [range]) & \\
Cardiovascular risk factors, n $(\%)$ & $31(62.0)$ \\
$\bullet \quad$ Diabetes mellitus & $38(76.0)$ \\
$\bullet \quad$ Hypertension & $35(70.0)$ \\
$\bullet \quad$ Hypercholesterolaemia & $9(18.0)$ \\
$\bullet \quad$ Current smoker & $32(64.0)$ \\
Medical history of family members, n $(\%)$ & $38(76.0)$ \\
$\bullet \quad$ Diabetes mellitus & $39(78.0)$ \\
$\bullet \quad$ Hypertension & $46(92.0)$ \\
\hline$\quad$ Hypercholesterolemia & Coronary artery disease
\end{tabular}

Values are Mean \pm SD [range] or number (percent).
Table II: Diagnostic accuracy of 64-slice CT coronary angiography for detecting $\leq 50 \%,>50 \%$ and $>75 \%$ stenoses in coronary arteries

\begin{tabular}{|c|c|c|c|}
\hline & $\leq 50 \%$ stenoses & $>50 \%$ stenoses & $>75 \%$ stenoses \\
\hline \multicolumn{4}{|l|}{ LM } \\
\hline - Sensitivity & $100.0(36.8-100.0)$ & $75.0(38.1-75.0)$ & $100.0(38.9-100.0)$ \\
\hline - Specificity & $93.8(91.1-93.8)$ & $100.0(96.8-100.0)$ & $97.9(95.4-97.9)$ \\
\hline - Accuracy & $94.0(88.9-94.0)$ & $98.0(92.1-98.0)$ & $98.0(93.1-98.0)$ \\
\hline - PPV & $40.0(14.7-40.0)$ & $100.0(50.8-100.0)$ & $66.7(26.0-66.7)$ \\
\hline - NPV & $100.0(97.2-100.0)$ & $97.9(94.7-97.9)$ & $100.0(97.4-10.0)$ \\
\hline \multicolumn{4}{|l|}{ Prox LAD } \\
\hline - Sensitivity & $100.0(75.5-100.0)$ & $100.0(89.0-100.0)$ & $81.1(63.1-81.8)$ \\
\hline - Specificity & $90.2(84.9-90.2)$ & $100.0(94.8-100.0)$ & $100.0(94.7-100.0)$ \\
\hline - Accuracy & $92.0(83.2-92.0)$ & $100.0(92.9-100.0)$ & $96.0(87.7-96.0)$ \\
\hline - PPV & $69.2(52.3-69.2)$ & $100.0(89.0-100.0)$ & $100.0(77.1-100.0)$ \\
\hline - NPV & $100.0(94.0-100.0)$ & $100.0(94.8-100.0)$ & $95.1(90.1-95.1)$ \\
\hline \multicolumn{4}{|l|}{ Mid LAD } \\
\hline - Sensitivity & $85.7(52.8-97.4)$ & $95.2(85.4-95.2)$ & $92.9(78.5-92.9)$ \\
\hline - Specificity & $86.0(80.7-87.9)$ & $100.0(92.9-100.0)$ & $100.0(94.4-100.0)$ \\
\hline - Accuracy & $86.0(76.8-89.3)$ & $98.0(89.8-98.0)$ & $98.0(90.0-98.0)$ \\
\hline - PPV & $50.0(30.8-56.8)$ & $100.0(89.7-100.0)$ & $100.0(84.5-100.0)$ \\
\hline - NPV & $97.4(91.3-99.5)$ & $96.7(89.8-96.7)$ & 97.3 (91.9-97.3) \\
\hline \multicolumn{4}{|l|}{ Distal LAD } \\
\hline - Sensitivity & $100.0(36.8-100.0)$ & $100.0(69.1-100.0)$ & $100.0(42.8-100.0)$ \\
\hline - Specificity & $93.8(91.1-93.8)$ & $100.0(96.6-100.0)$ & $100.0(97.6-100.0)$ \\
\hline - Accuracy & $94.0(88.9-94.0)$ & $100.0(93.8-100.0)$ & $100.0(95.4-100.0)$ \\
\hline - PPV & $40.0(14.7-40.0)$ & $100.0(69.1-100.0)$ & $100.0(42.8-100.0)$ \\
\hline - NPV & $100.0(97.2-100.0)$ & $100.0(96.6-100.0)$ & $100.0(97.6-100.0)$ \\
\hline \multicolumn{4}{|l|}{ Prox. LCX } \\
\hline - Sensitivity & $83.3(51.2-95.4)$ & $83.3(54.4-83.3)$ & $100.0(69.1-100.0)$ \\
\hline - Specificity & $97.7(93.3-99.4)$ & $100.0(96.0-100.0)$ & $100.0(96.6-100.0)$ \\
\hline - Accuracy & $96.0(88.3-98.9)$ & $98.0(91.0-98.0)$ & $100.0(93.8-100.0)$ \\
\hline - PPV & $83.3(51.2-95.4)$ & $100.0(65.2-100.0)$ & $100.0(69.1-100.0)$ \\
\hline - NPV & $97.7(93.3-99.4)$ & $97.8(93.9-97.8)$ & $100.0(96.6-100.0)$ \\
\hline \multicolumn{4}{|l|}{ Dist. LCX } \\
\hline - Sensitivity & $50.0(9.9-89.5)$ & $88.9(65.0-96.9)$ & $100.0(68.1-100.0)$ \\
\hline - Specificity & $95.8(94.2-97.5)$ & $97.6(92.3-99.3)$ & $95.5(91.1-95.5)$ \\
\hline - Accuracy & $94.0(90.8-97.2)$ & $96.0(87.4-98.9)$ & $96.0(88.3-96.0)$ \\
\hline - PPV & $33.3(6.6-59.7)$ & $88.9(65.0-96.9)$ & $75.0(51.1-75.0)$ \\
\hline - NPV & $97.9(96.2-99.6)$ & $97.6(92.3-99.3)$ & $100.0(95.4-100.0)$ \\
\hline \multicolumn{4}{|l|}{ Prox. RCA } \\
\hline - Sensitivity & $76.9(56.8-87.1)$ & $71.4(40.4-90.2)$ & $60.0(26.0-83.8)$ \\
\hline - Specificity & $94.6(87.5-98.2)$ & $93.0(88.0-96.1)$ & $95.6(91.8-98.2)$ \\
\hline - Accuracy & $90.0(79.5-95.3)$ & $90.0(81.3-95.3)$ & $92.0(85.2-96.8)$ \\
\hline PPV & $83.3(61.5-94.4)$ & $62.5(35.5-78.9)$ & $60.0(26.0-83.8)$ \\
\hline - NPV & $92.1(85.2-95.6)$ & $95.2(90.1-98.4)$ & $95.6(91.8-98.2)$ \\
\hline \multicolumn{4}{|l|}{ Mid. RCA } \\
\hline - Sensitivity & $75.0(33.8-94.9)$ & $86.7(70.4-92.0)$ & $84.6(66.2-90.8)$ \\
\hline - Specificity & $95.7(92.1-97.4)$ & $97.1(90.2-99.4)$ & $97.3(90.8-99.5)$ \\
\hline - Accuracy & $94.0(87.4-97.2)$ & $94.0(84.2-97.2)$ & $94.0(84.4-97.2)$ \\
\hline - PPV & $60.0(27.0-75.9)$ & $92.9(75.4-98.6)$ & $91.7(71.7-98.3)$ \\
\hline - NPV & $97.8(94.1-99.5)$ & $94.4(87.6-96.7)$ & $94.7(88.4-96.8)$ \\
\hline \multicolumn{4}{|l|}{ Dist. RCA } \\
\hline - Sensitivity & $100.0(37.5-100.0)$ & $75.0(48.4-85.0)$ & $62.5(36.5-72.6)$ \\
\hline - Specificity & $95.8(93.2-95.8)$ & $97.6(92.5-99.5)$ & $97.6(92.7-99.5)$ \\
\hline - Accuracy & $96.0(91.0-96.0)$ & $94.0(85.5-97.2)$ & $92.0(83.7-95.2)$ \\
\hline - PPV & $50.0(18.8-50.0)$ & $85.7(55.3-97.1)$ & $83.3(48.7-96.8)$ \\
\hline - NPV & $100.0(97.3-100.0)$ & $95.3(90.4-97.2)$ & $93.2(88.5-95.0)$ \\
\hline
\end{tabular}


Table III: Accuracy of 64-slice MDCT in the detection of stenosis in corresponding target areas on conventional angiogram

\begin{tabular}{lccrc}
\hline Tests with 95\% Cl & Observer 1 & Observer 2 & Kappa & Consensus \\
\hline $\begin{array}{l}\text { Detection of } \\
\text { stenosis } \leq 50 \%\end{array}$ & & & & \\
Sensitivity & $82.9(74.4-89.2)$ & $84.3(76.0-90.3)$ & 0.973 & $90.0(82.2-94.8)$ \\
Specificity & $96.8(96.0-97.4)$ & $96.9(96.2-97.5)$ & $96.5(95.8-97.0)$ \\
PPV & $69.9(62.7-75.2)$ & $71.1(64.1-76.2)$ & $70.0(64.0-73.8)$ \\
NPV & $98.4(97.7-99.0)$ & $98.8(97.8-99.1)$ & $99.1(98.4-99.5)$ \\
Accuracy & $95.6(94.2-96.7)$ & $95.9(94.5-96.9)$ & $96.0(94.7-96.8)$ \\
Detection of & & & \\
stenosis >50\% & & & \\
Sensitivity & $82.9(77.4-86.9)$ & $82.9(77.5-86.7)$ & 0.995 & $83.8(78.5-87.6)$ \\
Specificity & $98.2(97.4-98.8)$ & $98.4(97.6-99.0)$ & $98.4(97.6-99.0)$ \\
PPV & $87.6(81.9-91.8)$ & $88.5(82.7-92.6)$ & $88.6(83.0-92.6)$ \\
NPV & $97.4(96.6-98.0)$ & $97.5(96.7-98.0)$ & $97.6(96.8-98.2)$ \\
Accuracy & $96.2(94.8-97.3)$ & $96.4(95.0-97.4)$ & $96.5(95.1-97.5)$ \\
Detection of & & & \\
stenosis >75\% & & & \\
Sensitivity & $79.5(72.6-84.7)$ & $79.5(72.6-84.7)$ & 1.00 & $80.7(73.8-85.8)$ \\
Specificity & $98.3(97.6-98.9)$ & $98.3(97.6-98.9)$ & $98.3(97.6-98.9)$ \\
PPV & $83.5(76.2-89.0)$ & $83.5(76.2-89.0)$ & $83.8(76.6-89.0)$ \\
NPV & $97.8(97.0-98.4)$ & $97.8(97.0-98.4)$ & $97.9(97.2-98.5)$ \\
Accuracy & $96.5(95.1-97.5)$ & $96.5(95.1-97.5)$ & $96.6(95.2-97.6)$ \\
\hline & & &
\end{tabular}

Per artery analysis $(\mathrm{n}=850)$

Sensitivities for the detection of stenosis of $\leq 50 \%$ by observer 1 , observer 2 and consensus were $82.9 \%, 84.3 \%$, and $90.0 \%$, respectively, and specificities were $96.8 \%, 96.9 \%$, and $96.5 \%$ respectively and accuracies were $95.6 \%, 95.9 \%$, and $96.0 \%$ respectively

Sensitivities for the detection of stenosis of $>50 \%$ by observer 1 , observer 2 and consensus were $82.9 \%, 82.9 \%$, and $83.8 \%$, respectively, and specificities were $98.2 \%, 98.4 \%$, and $98.4 \%$ respectively and accuracies were $96.2 \%, 96.4 \%$, and $96.5 \%$ respectively.

Sensitivities for the detection of stenosis of $>75 \%$ by observer 1 , observer 2 and consensus were $79.5 \%, 79.5 \%$, and $80.7 \%$, respectively, and specificities were $98.3 \%, 98.3 \%$, and $98.3 \%$ respectively and accuracies were $96.5 \%, 96.5 \%$, and $96.6 \%$ respectively

\section{Discussion}

64-slice MDCT has a high discriminative power to detect obstructive coronary artery diseases in comparison with invasive coronary angiography as revealed in our finding.

To be a clinically useful tool for the diagnosis of patients with suspected CAD, complete visualization of all therapeutic relevant coronary arteries without excluding segments is necessary $y^{2}$. In the present study, we evaluated all arteries being $>1.5 \mathrm{~mm}$ in diameter, thereby finding a sensitivity and specificity of $80.7 \%$ and $98.3 \%$ for the detection of $>75 \%, 83.8 \%$ and $98.4 \%$ for the detection of $>50 \%$ and $90.0 \%$ and $96.5 \%$ for the detection of $\leq 50 \%$ coronary artery stenoses respectively. Similar to our study Niemann et $\mathrm{al}^{\mathbf{9}}$ reported a sensitivity of $81 \%$, a specificity of $97 \%$, a positive predictive value of $81 \%$ and a negative predictive value of $97 \%$ for detection of stenoses $>50 \%$ in 35 patients. Achenbach et al. ${ }^{10}$ found a sensitivity of $91 \%$ and a specificity of $84 \%$ in 64 patients.

Several other studies have compared the degree of stenosis detected by quantitative coronary angiography with that detected by 16 - or 64-slice CT. The overall correlation between 64 slice MDCT and invasive coronary angiography varies in different studies and appears to be moderate, even for selected segments with high image quality $^{\mathbf{1 1 - 1 4}}$. The sensitivities of 64-slice MDCT for the detection of stenosis of less than $50 \%$, stenosis of greater than $50 \%$, and stenosis of greater than $75 \%$ have been reported to be $79 \%, 73 \%$, and $80 \%$, respectively, and the specificity has been reported to be $97 \%$ in Leber et $\mathrm{al}^{\mathbf{1 5}}$.

Some recent studies have reported excellent diagnostic accuracy for 64-slice MDCT in the detection of significant stenosis in smaller coronary artery segments and side branches as well $(86 \%-$ $94 \%$ sensitivity and $93 \%-97 \%$ specificity) $)^{\mathbf{5 1 1}, \mathbf{1 5}, \mathbf{1 6}}$. In our study we evaluated small coronary vessels like obtuse marginal vessels and diagonal branches for stenosis. Our results to detect stenoses among these small vessels were also comparable with Raff et $\mathrm{al}^{11}$ findings.

Our study documents an excellent ability of 64 slice MDCT to rule out functionally relevant CAD as indicated by the high NPV. The high negative predictive values of $99.1 \%$ for the diagnosis of $\leq 50 \%$ stenosis, $97.6 \%$ for $>50 \%$ stenosis and $97.9 \%$ for $>75 \%$ stenosis in this study suggests an important future role of CT coronary angiography for reliably excluding $\mathrm{CAD}$ in patients with an equivocal clinical presentation, who may currently undergo a cost-extensive ICA. Our study result of high NPV was also comparable with some other studies $\mathbf{5 , 1 1 , 1 5 , 1 6}$ series. This high NPV was not affected by the different cut-offs for coronary stenosis chosen in this study $(\leq 50 \%,>50 \%$, or $>75 \%$ area stenosis).

On the other side an abnormal 64-slice CTA is a moderate predictor of functionally relevant coronary stenoses at $\leq 50 \%$ level ( $\mathrm{PPV}=70.0 \%$ ), strong predictor at $>50 \%$ level $(88.6 \%)$ and $>75.0 \%$ level $(83.8 \%)$ of coronary stenosis. Patients with positive CT angiogram need not always further evaluation for obstructive CAD and will not undergo invasive CA.

Conclusion: MDCT has reasonably high accuracy for detecting significant obstructive CAD when assessed at artery level. 


\section{Acknowledgment}

This study was supported by the DGHS, Mohakhali, Dhaka and Bangladesh College of Physicians and Surgeons, Mohakhali, Dhaka.

\section{References}

1. Hoffmann U, Ferencik M, Cury RC, Pena AJ, Coronary CT Angiography. J Nucl Med 2006; 47: 797-806.

2. Mollet NR, Cademartiri F, Nieman K, et al. Multislice spiral computed tomography coronary angiography in patients with stable angina pectoris. J Am Coll Cardiol 2004; 43: 2265-70.

3. Hoffmann U, Moselewski F, Cury RC, et al. Predictive value of 16-slice multidetector spiral computed tomography to detect significant obstructive coronary artery disease in patients at high risk for coronary artery disease: patient-versus segment-based analysis. Circulation 2004; 110: 2638-43.

4. Kuettner A, Beck T, Drosch $\mathrm{T}$, et al. Diagnostic accuracy of noninvasive coronary imaging using 16detector slice spiral computed tomography with $188 \mathrm{~ms}$ temporal resolution. J Am Coll Cardiol 2005; 45: 123 27.

5. Leschka S, Allkadhi H, Plass A, Desbiolles L, Gru'nenfelder J, Marincek B et al. Accuracy of MSCT coronary angiography with 64-slice technology:first experience. European Heart Journal 2005; 26: 1482-87.

6. Leber AW, Knez A, Ziegler Fv, Becker A, Nikolaou K, Paul $S$ et al. Quantification of Obstructive and Nonobstructive Coronary Lesions by 64-Slice Computed Tomography. A Comparative Study With Quantitative Coronary Angiography and Intravascular Ultrasound. JACC 2005; 46(1):147-54.

7. Achenbach S, Ropers D, Hoffmann U, et al. Assessment of coronary remodeling in stenotic and nonstenotic coronary atherosclerotic lesions by multidetector spiral computed tomography. J Am Coll Cardiol 2004; 43: 842-7.

8. Austen WG, Edwards JE, Frye RL, Gensini GG, Gott VL, Griffith LS, McGoon DC, Murphy ML, Roe BB.
A reporting system on patients evaluated for coronary artery disease. Report of the ad hoc committee for grading of coronary artery disease, council on cardiovascular surgery, American Heart Association. Circulation 1975; 51: 5-40.

9. Nieman K, Cademartiri F, Lemos PA. Non-invasive coronary angiography with fast submillimeter multislice spiral computed tomography. Circulation 2002; 106: 2051-4.

10. Achenbach S, Giesler $\mathrm{T}$, Ropers $\mathrm{D}$, Ulzheimer $\mathrm{S}$, Derlien H, Schulte C, et al. Detection of coronary artery stenoses by contrast-enhanced, retrospectively electrocardiographically-gated, multislice spiral computed tomography. Circulation 2001; 103: 2535 38 .

11. Raff GL, Gallagher MJ, O'Neill WW. Diagnostic accuracy of noninvasive coronary angiography using 64-slice spiral computed tomography. J Am Coll Cardiol. 2005; 46: 552-57.

12. Morgan-Hughes GJ, Roobottom CA, Owens PE, Marshall AJ. Highly accurate coronary angiography with submillimetre, 16 slice computed tomography. Heart. 2005; 91: 308-13.

13. Cury RC, Pomerantsev EV, Ferencik M. Comparison of the degree of coronary stenoses by multidetector computed tomography versus by quantitative coronary angiography. Am J Cardiol. 2005; 96: 784-87.

14. Kefer J, Coche E, Legros G. Head-to-head comparison of three dimensional navigator-gated magnetic resonance imaging and 16-slice computed tomography to detect coronary artery stenosis in patients. J Am Coll Cardiol. 2005; 46: 92-100.

15. Leber AW, Becker A, Knez A, von Ziegler F, Sirol M, Nikolaou K et al. Accuracy of 64-slice computed tomography to classify and quantify plaque volumes in the proximal coronary system: a comparative study using intravascular ultrasound. J Am Coll Cardiol. 2006; 47: 672-77.

16. Mollet NR, Cademartiri F, van Mieghem CA. Highresolution spiral computed tomography coronary angiography in patients referred for diagnostic conventional coronary angiography. Circulation 2005; 112: $2318-23$. 